PROCEEDINGS OF THE

AMERICAN MATHEMATICAL SOCIETY

Volume 132, Number 12, Pages 3641-3651

S 0002-9939(04)07506-9

Article electronically published on May 20, 2004

\title{
THE INDEPENDENCE OF CHARACTERS ON NON-ABELIAN GROUPS
}

\author{
DAVID GROW AND KATHRYN E. HARE
}

(Communicated by Andreas Seeger)

\begin{abstract}
We show that there are characters of compact, connected, nonabelian groups that approximate random choices of signs. The work was motivated by Kronecker's theorem on the independence of exponential functions and has applications to thin sets.
\end{abstract}

\section{INTRODUCTION}

Kronecker's theorem states that if $x_{1}, \ldots, x_{k}$ are real numbers that are linearly independent over $\mathbb{Q}$, then for any $\varepsilon>0$ and $\alpha_{1}, \ldots, \alpha_{k} \in \mathbb{R}$ there exists a real number $y$ with $\left|e^{i y x_{j}}-e^{i \alpha_{j}}\right|<\varepsilon$ for $j=1, \ldots, k$. This classical theorem has inspired many mathematicians since it was first proved more than 100 years ago, and has been extended in many ways. For example, Bohr [1], 2] exploited the idea to study the absolute convergence of Fourier series of almost periodic functions, Hewitt and Zuckerman [11] generalized the theorem to deduce various approximation theorems, and Rudin [19] introduced the related notion of a Kronecker set.

A variation of Kronecker's theorem on the independence of exponential functions states that there is an infinite set of integers $\left\{n_{k}\right\}$ with the property that for any choice of signs $\left\{r_{j}\right\}$ there is some $x \in T$ with $\sup _{j}\left|e^{i n_{j} x}-r_{j}\right|<1$. A similar result is true for all infinite, compact abelian groups [9].

In this paper we study the independence properties of characters of compact, connected, non-abelian groups. We prove that for all such groups there are infinite sets of characters that approximate random choices of signs $\left\{r_{k}\right\}$. We do this first for Lie groups where the characters are well understood, and then appeal to the structure theorem for compact, connected groups.

Since the value of a character, $\chi_{k}$, can be as large as its degree, one might expect that an arbitrary choice of signs could be approximated by $\left\{\chi_{k}(x) / \operatorname{deg} \chi_{k}\right\}$. It is not possible to do this in compact, simple Lie groups (see [16], 18]); however, we are able to show that for most such groups one can approximate arbitrary choices of signs by $\left\{\chi_{k}(x) /\left(\operatorname{deg} \chi_{k}\right)^{a}\right\}$ for some $a>0$.

An application is given to the study of thin sets in compact, non-abelian groups.

Received by the editors August 22, 2003.

2000 Mathematics Subject Classification. Primary 43A65; Secondary 43A46, 22E46.

Key words and phrases. Characters, independence, compact non-abelian groups, compact Lie groups.

This research was supported in part by NSERC and the Swedish Natural Sciences Research Council.

(C)2004 American Mathematical Society 


\section{INDEPENDENCE OF CHARACTERS}

2.1. Independence of characters of compact, abelian groups. In [9] Hartman and Ryll-Nardzewski showed that the dual of every infinite, compact abelian group contains an infinite set of characters $\left\{\chi_{j}\right\}$ such that for any choice of signs $\left\{r_{j}\right\}$ there is some $x$ in the group satisfying $\sup _{j}\left|\chi_{j}(x)-r_{j}\right|<1$. The goal of this paper is to extend this result to any compact, connected group $G$. The dual of $G$, denoted $\widehat{G}$, is a maximal set of inequivalent, irreducible, unitary representations of $G$. The irreducible representations of compact groups are always finite dimensional and their traces are the characters of $G$.

Our main result is:

Theorem 2.1. Let $G$ be any infinite, compact, connected group. There is an infinite set of representations $\left\{\lambda_{j}\right\} \subset \widehat{G}$ such that for all choices of signs $\left\{r_{j}\right\}$ there is some $x \in G$ with

$$
\sup _{j}\left|\operatorname{Tr} \lambda_{j}(x)-r_{j}\right|<1 .
$$

We begin by considering compact, connected, simply connected, simple Lie groups because explicit formulas are known for their characters. Once the independence properties for characters on these groups have been established it is not difficult to use abelian group theory and the structure theorem to transfer the results for compact, Lie groups to all compact, connected groups.

2.2. Notation. Let $G$ denote a compact, connected, simply connected, simple Lie group of rank $n$, and let $T$ be a maximal torus of $G$ associated with a system of positive roots $\Phi^{+}$. If $\mathfrak{h}$ is a Cartan subalgebra of the Lie algebra $\mathfrak{g}$ of $G$ corresponding to $T$, then $T=\{\exp (X): X \in \mathfrak{h}\}$. Pick and fix a basis $\left\{H_{1}, \ldots, H_{n}\right\}$ for $\mathfrak{h}$ such that each $\alpha\left(H_{k}\right)$ is an integer for all $\alpha \in \Phi^{+}$(cf. [20] p. 181]). The coordinates of $X \in \mathfrak{h}$ are the unique real numbers $\xi_{1}, \ldots, \xi_{n}$ such that $X=\sum_{i=1}^{n} \xi_{i} H_{i}$. The Weyl character formula (cf. 21]) specifies the value of a character on the torus and, since a character is a class function, this uniquely determines it. For more details and further background information on the structure and representation theory of compact, Lie groups the reader is referred to [12] and [20].

Of primary interest to us are the representations $\sigma_{N}$ on $G$ whose characters on the torus are given by the Weyl character formula as

$$
\operatorname{Tr} \sigma_{N}(x) \equiv \frac{D_{N}(x)}{D(x)} \text { where } D_{N}(\exp (X))=\prod_{\alpha \in \Phi^{+}} \sin (N \alpha(X) / 2) \text { and } D=D_{1} .
$$

(This formula is understood as a limit if $D(x)=0$.) Our study of these particular representations was motivated in part by the work of Dooley who showed in [4] that the set of representations $\left\{\sigma_{N_{j}}\right\}$, for $N_{j}$ growing sufficiently rapidly, had the special feature of being a central $p$-Sidon set for $p>1$. Later in this section we will prove that these characters have the desired independence property.

2.3. Periodicity properties. The key to our approximation results is the periodicity of the functions $D_{N}$. In particular, one should note that $D_{N}(\exp (X))=$ $D_{N}(\exp (Y))$ if each coordinate of $X-Y$ is an integer multiple of $4 \pi / N$. In the first two lemmas we derive useful consequences of this periodicity. 
Lemma 2.2. Let $\left\{B_{j}\right\}_{j=1}^{J}$ be a finite collection of non-empty, open subsets of $T$. Then there is an integer $N_{0}$ such that for any $N \geq N_{0}$, the range of $D_{N}$ restricted to any of the sets $B_{j}$ is the same as the range of $D$ on $T$.

Proof. The range of $D_{N}$ and the range of $D$ clearly coincide. Thus it suffices to show that $D_{N}$ and $\left.D_{N}\right|_{B_{j}}$ have the same range.

Fix elements

$$
x_{j}=\exp \left(\sum_{i=1}^{n} \xi_{i}^{(j)} H_{i}\right) \in B_{j}, j=1, \ldots, J,
$$

and choose an integer $N_{0}$ such that if $\left|\xi_{i}^{(j)}-\zeta_{i}^{(j)}\right|<4 \pi / N_{0}$ for all $i$ and all $j$, then $\exp \left(\sum_{i=1}^{n} \zeta_{i}^{(j)} H_{i}\right) \in B_{j}$.

Let $N \geq N_{0}$. Given $y=\exp \left(\sum_{i=1}^{n} v_{i} H_{i}\right) \in T$, select integers $l_{j}(i), i=1, \ldots, n$, $j=1, \ldots, J$ satisfying

$$
\max _{i}\left\{\left|\xi_{i}^{(j)}-\left(v_{i}-4 \pi l_{j}(i) / N\right)\right|\right\}<4 \pi / N .
$$

Therefore, if

$$
Z_{j}=\sum_{i=1}^{n}\left(v_{i}-4 \pi l_{j}(i) / N\right) H_{i}
$$

then $\exp \left(Z_{j}\right) \in B_{j}$, and the remarks above imply that $D_{N}\left(\exp \left(Z_{j}\right)\right)=D_{N}(y)$. Thus the range of $D_{N}$ on $B_{j}$ contains the range of $D_{N}$.

Lemma 2.3. Let $c \neq 0$ and assume $\pm c \in$ Range $D$. Let $\delta>0$ and let $B \subset T$ be any non-empty, open set. Then there is an increasing sequence of integers $\left\{N_{k}\right\}$ such that for any choice of signs $\left\{r_{k}\right\}$ there exists $z \in B$ with

$$
\left|D_{N_{k}}(z)-r_{k} c\right|<\delta \text { for all } k \text {. }
$$

Proof. Apply Lemma 2.2 to choose $N_{1}$ such that the range of $D_{N_{1}}$ restricted to $B$ includes both $\pm c$, say $D_{N_{1}}\left(x_{w_{1}}\right)=w_{1} c$ for $x_{w_{1}} \in B, w_{1}= \pm 1$. Because $D_{N}$ is continuous, we can choose closed neighbourhoods $B_{w_{1}}$ in $B$, such that $x_{w_{1}} \in \operatorname{int} B_{w_{1}}$ and having the property that for all $z \in B_{w_{1}}$,

$$
\left|D_{N_{1}}(z)-w_{1} c\right|=\left|D_{N_{1}}(z)-D_{N_{1}}\left(x_{w_{1}}\right)\right|<\delta .
$$

Without loss of generality, we may assume the diameter of $B_{w_{1}}$ is less than $1 / 2$.

Now assume inductively that for $w_{j}= \pm 1, j=1, \ldots, k$ we have found points $x_{w_{1} \cdots w_{k}}$, closed neighbourhoods

$$
B_{w_{1} \cdots w_{k}} \subset \operatorname{int} B_{w_{1} \cdots w_{k-1}}
$$

and an increasing sequence of integers $N_{1}, \ldots, N_{k}$ such that $\operatorname{diam} B_{w_{1} \cdots w_{k}}<1 / 2^{k}$, $x_{w_{1} \cdots w_{k}} \in \operatorname{int} B_{w_{1} \cdots w_{k}}, D_{N_{k}}\left(x_{w_{1} \cdots w_{k}}\right)=w_{k} c$, and

$$
\left|D_{N_{k}}(z)-w_{k} c\right|<\delta \text { for all } z \in B_{w_{1} \cdots w_{k}} .
$$

From Lemma 2.2 we may choose $N_{k+1}>N_{k}$ such that the range of $D_{N_{k+1}}$ restricted to each of the (finitely many) sets int $B_{w_{1} \cdots w_{k}}$ for $w_{j}= \pm 1, j=1, \ldots, k$, contains both $\pm c$. Indeed, suppose that for $w_{k+1}= \pm 1, x_{w_{1} \cdots w_{k+1}} \in \operatorname{int} B_{w_{1} \cdots w_{k}}$ satisfies

$$
D_{N_{k+1}}\left(x_{w_{1} \cdots w_{k+1}}\right)=w_{k+1} c
$$


Then obtain closed neighbourhoods $B_{w_{1} \cdots w_{k+1}}$ of $x_{w_{1} \cdots w_{k+1}}$, contained in int $B_{w_{1} \cdots w_{k}}$, so that for all $z \in B_{w_{1} \cdots w_{k+1}}$,

$$
\left|D_{N_{k+1}}(z)-w_{k+1} c\right|<\delta
$$

Certainly we can achieve this with a diameter of $B_{w_{1} \cdots w_{k+1}}$ less than $1 / 2^{k+1}$. This completes the induction step.

To see that the sequence $\left\{N_{k}\right\}$ has the required property take any choice of signs $\left\{r_{k}\right\}_{k=1}^{\infty}$ and consider the sets $\left\{B_{r_{1} \cdots r_{k}}\right\}_{k=1}^{\infty}$. These sets are non-empty, nested, compact and shrinking to zero in diameter; hence there exists a unique $z \in \bigcap_{k} B_{r_{1} \cdots r_{k}} \subset$ $B$. Since $z \in B_{r_{1} \cdots r_{k}}$ for every $k$, it follows by construction that $\left|D_{N_{k}}(z)-r_{k} c\right|<\delta$ for all $k$.

2.4. Independence of characters of Lie groups. We are now ready to prove that if $\left\{N_{j}\right\}$ grows sufficiently quickly, then the characters $\left\{\operatorname{Tr}_{N_{j}}\right\}$ can be used to approximate arbitrary choices of signs.

Theorem 2.4. Let $G$ be a compact, simple, simply connected, connected Lie group. For every $\varepsilon>0$ there is a set of representations $\left\{\lambda_{j}\right\}_{j=1}^{\infty} \subset \widehat{G}$ such that for any choice of signs $\left\{r_{j}\right\}$ there is some $z \in G$ with

$$
\left|\operatorname{Tr} \lambda_{j}(z)-r_{j}\right|<\varepsilon \text { for all } j .
$$

Proof. Without loss of generality, we may assume $\varepsilon<1$. Choose $g \in T$ such that $D(g) \neq 0$. It is well known that $D$ is alternating, i.e., $D(x)=\operatorname{det} w D(w(x))$ for any $w$ in the Weyl group of $G([21,4.14])$. Thus $D$ takes on both the (non-zero) values $\pm D(g)$.

Let $d=|D(g)|$. Choose a closed neighbourhood $B$ of $g$ such that for all $y \in B$, $|D(y)-D(g)|<\varepsilon d / 4$. Then apply Lemma 2.3, taking $c=D(g)$ and $\delta=\varepsilon d / 4$, to choose a sequence $\left\{N_{k}\right\}$ with the outlined properties. Set $\lambda_{k}=\sigma_{N_{k}}$.

Given a choice of signs $\left\{r_{k}\right\}$ select $z \in B$ such that $\left|D_{N_{k}}(z)-r_{k} c\right|<\delta$ for all $k$. Since $z \in B,|D(z)-D(g)|<\varepsilon d / 4$ and hence

$$
\left|\operatorname{Tr} \lambda_{k}(z)-r_{k}\right| \leq\left|\frac{D_{N_{k}}(z)}{D(z)}-r_{k} \frac{D(g)}{D(z)}\right|+\left|\frac{D(g)}{D(z)}-1\right|<\varepsilon
$$

for all $k$.

2.5. Approximation of signs for compact, connected groups. We are now ready to show that there are infinite, "independent" sets of characters in any compact, connected group.

Proof of Theorem 2.1. By the structure theorem ([15, 6.5.6]) we may assume $G$ is $\mathfrak{T} \times \prod_{i \in I} G_{i} / K$ where the $G_{i}$ are compact, simple, simply connected, connected Lie groups, $\mathfrak{T}$ is a compact, abelian group, $K$ is a subset of the centre of $\mathfrak{T} \times \prod_{i \in I} G_{i}$ and $K \cap \mathfrak{T}$ is the identity of $G$. Since the result is known for abelian groups we may assume $I$ is non-empty.

Choose any index $i_{0} \in I$ and consider the representations $\sigma_{N}$ on $G_{i_{0}}$, with $N$ odd. A fact about root systems is that $z=\exp (Z)$ belongs to the centre of $G_{i_{0}}$ if and only if $\alpha(Z) \in 2 \pi \mathbb{Z}$ for all positive roots $\alpha$ of $G_{i_{0}}$ ([3, V.2]). Thus one can verify from the formula for $\operatorname{Tr} \sigma_{N}$ (2.1) that if $z$ is in the centre and $N$ is odd, then $\operatorname{Tr} \sigma_{N}(z)=\operatorname{deg} \sigma_{N}$ and therefore $\sigma_{N}(z)=I_{d\left(\sigma_{N}\right)}$. Since $\left(t,\left(g_{i}\right)\right)$ belongs to the centre of $\mathfrak{T} \times \prod_{i \in I} G_{i}$ if and only if $g_{i} \in$ centre $G_{i}$ for each $i$, it follows that the 
homomorphism $\lambda_{N}$ defined on $G$ by $\lambda_{N}\left(\left(t,\left(g_{i}\right)\right) K\right) \equiv \sigma_{N}\left(g_{i_{0}}\right)$ is well defined. The reader can easily check that the representations $\lambda_{N}$ are irreducible and inequivalent.

From (the proof of) Theorem[2.4 we can obtain a sequence of integers $\left\{N_{j}\right\}$ such that given any choice of signs $\left\{r_{j}\right\}$ there is some $x \in G_{i_{0}}$ with

$$
\sup _{j}\left|\operatorname{Tr} \sigma_{N_{j}}(x)-r_{j}\right|<1 \text {. }
$$

The corresponding representations $\left\{\lambda_{N_{j}}\right\}$ interpolate at the coset with representative $\left(e, g_{i}\right)$ where $g_{i_{0}}=x$ and $g_{i}=e$ otherwise.

\section{Better Approximations on Lie Groups}

For any representation $\lambda$ we have $\operatorname{Tr} \lambda(e)=\operatorname{deg} \lambda$; thus one might speculate that it would be possible to find an infinite set of representations $\left\{\lambda_{j}\right\}$ such that for all choices of signs $\left\{r_{j}\right\}$ there is some $x \in G$ with

$$
\sup _{j}\left|\frac{\operatorname{Tr} \lambda_{j}(x)}{\operatorname{deg} \lambda_{j}}-r_{j}\right|<1 .
$$

This is not true for compact, connected, simple Lie groups, however, because it is known that $\operatorname{Tr} \lambda(x) / \operatorname{deg} \lambda \rightarrow 0$ off the centre of the group ([16], 18]) and the centre of such a group is finite.

But characters can interpolate larger values than just signs in most simple Lie groups. To prove this we need to generalize Lemmas 2.2 and 2.3 It will be convenient to introduce some additional notation.

3.1. Notation. As usual, $W$ will denote the Weyl group of $G$. For each $g=$ $\exp (Y) \in T$, it will be useful to consider the following subset of the positive roots

$$
\Phi^{+}(g)=\left\{\alpha \in \Phi^{+}: \alpha(Y) \in 2 \pi \mathbb{Z}\right\},
$$

and the related subset of the torus

$$
X(g)=\left\{y \in T: \Phi^{+}(g)=\Phi^{+}(y)\right\} .
$$

The (dense) elements $g$ of the torus for which $\Phi^{+}(g)$ is empty are known as regular. We also need to consider the function on $T$ given by

$$
D^{g}(\exp (X))=\prod_{\alpha \in \Phi^{+} \backslash \Phi^{+}(g)} \sin (\alpha(X) / 2) .
$$

Lemma 3.1. Let $g \in T$ and suppose there exists $w \in W$ that fixes $\Phi^{+}(g)$. Then $\operatorname{det} w D^{g}(x)=D^{g}(w(x))$ for all $x \in T$.

Proof. Since $D$ is alternating, we have for $x=\exp (X)$ that

$$
\begin{aligned}
D(x) & =\prod_{\alpha \in \Phi^{+}(g)} \sin (\alpha(X) / 2) D^{g}(x) \\
& =\operatorname{det} w \prod_{\alpha \in \Phi^{+}(g)} \sin \left(\alpha\left(A d\left(g_{w}\right) X\right) / 2\right) D^{g}(w(x))
\end{aligned}
$$

where $w(y)=g_{w} y g_{w}^{-1}$ for all $y \in T$. Since $w$ fixes $\Phi^{+}(g)$, this implies that

$$
D(x)=\operatorname{det} w \prod_{\alpha \in \Phi^{+}(g)} \sin (\alpha(X) / 2) D^{g}(w(x)) .
$$


When $x$ is regular, $\prod_{\alpha \in \Phi^{+}(g)} \sin (\alpha(X) / 2) \neq 0$, and hence $D^{g}(x)=\operatorname{det} w D^{g}(w(x))$. Because such $x$ are dense in $T$, and the functions $D^{g}$ and $D^{g} \circ w$ are continuous, the same equality holds for all $x \in T$.

Next, we generalize Lemma 2.2. We will write $D_{N}^{g}$ for the function $\exp (X) \mapsto$ $\prod_{\alpha \in \Phi^{+} \Phi^{+}(g)} \sin (N \alpha(X) / 2)$ and denote by $\delta_{i}, 1 \leq i \leq n$, the coordinate projections on $\mathfrak{h}$ with respect to the basis $\left\{H_{1}, \ldots, H_{n}\right\}$.

Lemma 3.2. Fix $k \in \mathbb{N} \backslash\{1\}$ and suppose $\Phi^{+}(g)=\left\{\delta_{i}-\delta_{j}: 1 \leq i<j \leq k\right\}$. Let $\left\{B_{j}\right\}_{j=1}^{J}$ be a finite collection of open subsets of $T$ with $B_{j} \cap X(g)$ non-empty for each $j$. Then there is an integer $N$ such that the range of $D_{N}^{g}$ restricted to each set $B_{j} \cap X(g)$ contains the range of $D^{g}$ on $X(g)$.

Proof. Let $z \in X(g)$ and consider $D^{g}(z)$. The structure of $\Phi^{+}(g)$ guarantees that we may assume $z=\exp (Z)$ where $Z=\sum_{j=1}^{n} \zeta_{j} H_{j}$ with $\zeta_{1}=\cdots=\zeta_{k}$ and $\alpha(Z) \notin 2 \pi \mathbb{Z}$ for all $\alpha \notin \Phi^{+}(g)$. Consequently, $\exp (Z / N) \in X(g)$, and hence the range of $D_{N}^{g}$ restricted to $X(g)$ contains the range of $\left.D^{g}\right|_{X(g)}$.

Fix any $x_{j} \in B_{j} \cap X(g), j=1, \ldots, J$. The continuity of exp and the functions $\alpha \in \Phi^{+}$ensure that if $z_{j}=\exp \left(Z_{j}\right)$ is sufficiently close to $x_{j}$, then $\alpha\left(Z_{j}\right) \notin 2 \pi \mathbb{Z}$ for all $\alpha \notin \Phi^{+}(g)$. Thus there is some $\varepsilon>0$ such that if $z_{j}=\exp \left(Z_{j}\right) \in T$, $\left\|x_{j}-z_{j}\right\|<\varepsilon$, and the first $k$ coordinates of $Z_{j}$ are all equal, then $z_{j} \in B_{j} \cap X(g)$.

Choose $N>4 \pi / \varepsilon$ and write $x_{j}=\exp \left(X_{j}\right)$ where $X_{j}=\sum_{i=1}^{n} \xi_{i}^{(j)} H_{i}, j=$ $1, \ldots, J$. Given $y=\exp \left(\sum_{i=1}^{n} v_{i} H_{i}\right) \in X(g)$, select integers $l_{j}(i), i=1, \ldots, n$, with $l_{j}(1)=\cdots=l_{j}(k)$ and

$$
\max _{i}\left\{\left|\xi_{i}^{(j)}-\left(v_{i}-4 \pi l_{j}(i) / N\right)\right|\right\}<4 \pi / N<\varepsilon .
$$

If $z_{j} \in T$ is defined by

$$
z_{j}=\exp \left(\sum_{i=1}^{n}\left(v_{i}-4 \pi l_{j}(i) / N\right) H_{i}\right) \text { for } j=1, \ldots, J,
$$

then the remarks above establish that $z_{j} \in B_{j} \cap X(g)$ and $D_{N}^{g}\left(z_{j}\right)=D_{N}^{g}(y)$. Thus the range of $D_{N}^{g}$ on $B_{j} \cap X(g)$ contains the range of $D_{N}^{g}$ on $X(g)$, and consequently contains the range of $\left.D^{g}\right|_{X(g)}$.

Lemma 3.3. Let $c \neq 0$ and assume $\pm c \in$ Range $\left.D^{g}\right|_{X(g)}$. Let $\delta>0$ and let $B \subset T$ be any open set with $B \cap X(g)$ non-empty. Then there is an increasing sequence of integers $\left\{N_{k}\right\}$ such that for any choice of signs $\left\{r_{k}\right\}$ there exists $z \in B$ with $\Phi^{+}(z) \supseteq \Phi^{+}(g)$ and

$$
\left|D_{N_{k}}^{g}(z)-r_{k} c\right|<\delta \text { for all } k \text {. }
$$

Proof. This is a routine generalization of Lemma 2.3 using the lemma above. The fact that the element $z$, found through the nested sets construction, is a limit of $\left\{x_{r_{1} \cdots r_{k}}\right\} \subset X(g)$ ensures that $\Phi^{+}(z) \supseteq \Phi^{+}(g)$.

We can now improve Theorem 2.4

Theorem 3.4. Let $G$ be a compact, simple, simply connected, connected Lie group, other than $S U(2)$ or $S U(3)$. For every $\varepsilon>0$ there is an infinite set of representations $\left\{\lambda_{j}\right\} \subset \widehat{G}$ with the property that for all choices of signs $\left\{r_{j}\right\}$ there is some 
$x \in G$ with

$$
\left|\frac{\operatorname{Tr} \lambda_{j}(x)}{\operatorname{deg} \lambda_{j}}-\frac{r_{j}}{\operatorname{deg} \lambda_{j}^{s}}\right|<\frac{\varepsilon}{\operatorname{deg} \lambda_{j}^{s}} \text { for all } j,
$$

for some $s=s(G)<1$.

Remark 3.1. (1) Every element in $S U(2)$ (Lie type $A_{1}$ ) is either central or regular. It can be easily seen from the Weyl character formula that for all regular $x$ and representations $\lambda,|\operatorname{Tr} \lambda(x)| \leq c(x)$. Since the centre of $S U(2)$ is finite, this shows that the theorem cannot hold for $S U(2)$.

(2) For $S U(3)$ (Lie type $A_{2}$ ) there are elements neither central nor regular, namely those of the form $g=\exp (\operatorname{diag}(i x, i x,-2 i x))$ or a "permutation" thereof. However, all such $g$ satisfy $D^{g}(g)>0$, and consequently $\operatorname{Tr} \sigma_{N}(g) \geq 0$. This means it is impossible to do the desired approximation with these representations (which is what we show for all the other groups). It is possible to obtain the desired approximation on $S U(3)$ by using a different family of representations, but this is much more technical and the argument is not included in this paper.

Proof. The basic strategy of the proof will be to show that there exists some $g \in T$ and $w$ in the Weyl group with negative determinant, such that $\Phi^{+}(g)$ is fixed by $w$ and has the form

for some $k>1$.

$$
\Phi^{+}(g)=\left\{\delta_{i}-\delta_{j}: 1 \leq i<j \leq k\right\}
$$

Once this is established we will argue in a similar manner to the proof of Theorem 2.4. but appealing to the more general lemmas above, as follows: First, because $w$ fixes $\Phi^{+}(g), w(g) \in X(g)$ and thus an application of Lemma 3.1 shows that $\left.D^{g}\right|_{X(g)}$ contains the two (non-zero) values $\pm D^{g}(g)$. Let $d=\left|D^{g}(g)\right|$. Choose a closed neighbourhood $B$ of $g$ such that for all $y \in B,\left|D^{g}(y)-D^{g}(g)\right|<\varepsilon d / 4$. Apply Lemma 3.3, taking $c=D^{g}(g)$ and $\delta=\varepsilon d / 4$, to obtain a sequence of integers $\left\{N_{k}\right\}$ with the outlined properties. We take $\lambda_{k}=\sigma_{N_{k}}$.

Given a choice of signs $\left\{r_{k}\right\}$ choose $z \in B$ such that $\Phi^{+}(z) \supseteq \Phi^{+}(g)$ and $\left|D_{N_{k}}^{g}(z)-r_{k} c\right|<\delta$ for all $k$. Thus

$$
\left|\frac{D_{N_{k}}^{g}(z)}{D^{g}(z)}-r_{k}\right| \leq\left|\frac{D_{N_{k}}^{g}(z)}{D^{g}(z)}-r_{k} \frac{D^{g}(g)}{D^{g}(z)}\right|+\left|\frac{D^{g}(g)}{D^{g}(z)}-1\right|<\varepsilon \text { for all } k .
$$

The fact that $z \in B$ ensures that $D^{g}(z) \neq 0$ and consequently $\Phi^{+}(z)=\Phi^{+}(g)$. Thus

$$
\begin{aligned}
\operatorname{Tr} \lambda_{j}(z) & =\lim _{x \rightarrow z, x \text { regular }} \prod_{\alpha \in \Phi^{+}(g)} \frac{\sin \left(N_{j} \alpha(X) / 2\right)}{\sin (\alpha(X) / 2)} \prod_{\alpha \in \Phi^{+} \backslash \Phi^{+}(g)} \frac{\sin \left(N_{j} \alpha(Z) / 2\right)}{\sin (\alpha(Z) / 2)} \\
& =N_{j}^{\left|\Phi^{+}(g)\right|} \frac{D_{N_{j}}^{g}(z)}{D^{g}(z)} .
\end{aligned}
$$

Let $s=1-\frac{\left|\Phi^{+}(g)\right|}{\left|\Phi^{+}\right|}$. Since $\operatorname{deg} \lambda_{j}=N_{j}^{\left|\Phi^{+}\right|}$we obtain

$$
\left|\frac{\operatorname{Tr} \lambda_{j}(z)}{\operatorname{deg} \lambda_{j}}-\frac{r_{j}}{\left(\operatorname{deg} \lambda_{j}\right)^{s}}\right|=\frac{1}{N_{j}^{\left|\Phi^{+}\right|-\left|\Phi^{+}(g)\right|}}\left|\frac{D_{N_{k}}^{g}(z)}{D^{g}(z)}-r_{j}\right|<\frac{\varepsilon}{\left(\operatorname{deg} \lambda_{j}\right)^{s}}
$$

as desired.

To establish the existence of such $g \in G$ and $w \in W$, each classical Lie type can be considered separately. For example, in type $B_{n}, n \geq 3$, a basis for $\mathfrak{h}$ is the set 
of $2 n+1$ by $2 n+1$ matrices $\left\{H_{1}, \ldots, H_{n}\right\}$ where $H_{k}=\left[a_{r s}\right]$ with $a_{2 k-1,2 k}=1$, $a_{2 k, 2 k-1}=-1$, and all other $a_{r s}=0$. Take

$$
g=\exp \left(y H_{n}+\sum_{j=1}^{n-1} x H_{j}\right)
$$

in $T$ with $x$ and $y$ chosen so that $\Phi^{+}(g)=\left\{\delta_{i}-\delta_{j}: 1 \leq i<j \leq n-1\right\}$; the element $w$ that corresponds to the simple change of sign in the $n$th coordinate on $\mathfrak{h}$ fixes $\Phi^{+}(g)$ and is odd. For type $A_{n}$ with $n \geq 3$, an analogous argument produces $g \in T$ with $\Phi^{+}(g)=\left\{\delta_{i}-\delta_{j}: 1 \leq i<j \leq n-1\right\}$. In this case, let $w$ correspond to the transposition $\operatorname{diag}\left(z_{1}, \ldots, z_{n-1}, z_{n}, z_{n+1}\right) \mapsto \operatorname{diag}\left(z_{1}, \ldots, z_{n-1}, z_{n+1}, z_{n}\right)$ in $\mathfrak{h}$. Types $C_{n}, D_{n}, E_{n}$ and $F_{4}$ are similar.

For $B_{2}$ and $G_{2}$ the argument is slightly different. In the case of $B_{2}$, a basis for $\mathfrak{h}$ is the set of 5 by 5 matrices $\left\{H_{1}, H_{2}\right\}$ where $H_{k}=\left[a_{r s}\right]$ with $a_{2 k-1,2 k}=1$, $a_{2 k, 2 k-1}=-1$, and all other $a_{r s}=0$. We take $g=\exp \left(x H_{1}+x H_{2}\right)$ so that $\Phi^{+}(g)=\left\{\delta_{1}-\delta_{2}\right\}$. Notice that $D^{g}(g)=\sin ^{2}(x / 2) \sin (x)=-D^{g}\left(g^{-1}\right)$. This change of sign allows similar arguments to be applied. Type $G_{2}$ is analogous.

\section{Applichtions to thin sets}

In this section we will be studying subsets of $\widehat{G}$ with particular properties. To define these properties we need to introduce some normed spaces: First, given a matrix $A$ we let $\|A\|_{\infty}$ denote the largest eigenvalue of $|A|$. For $E \subset \widehat{G}$ let

$$
l_{\infty}(E)=\left\{\left(A_{\sigma}\right)_{\sigma \in E}: A_{\sigma} \text { is a } \operatorname{deg}(\sigma) \times \operatorname{deg}(\sigma) \text { matrix with } \sup _{\sigma}\left\|A_{\sigma}\right\|_{\infty}<\infty\right\},
$$

and let $l_{\infty}^{z}(E)$ denote the subset of $l_{\infty}(E)$ containing the matrices of the form $\left(c_{\sigma} I_{\operatorname{deg} \sigma}\right)_{\sigma \in E}$. For $\phi \in l_{\infty}(E)$ and $a \geq 0$ we let

$$
\|\phi\|_{a, \infty}=\sup \left\{(\operatorname{deg} \sigma)^{1-a}\|\phi(\sigma)\|_{\infty}: \sigma \in E\right\}
$$

and denote by $l_{a, \infty}(E)$ (respectively, $\left.l_{a, \infty}^{z}(E)\right)$ the set of all $\phi \in l_{\infty}(E)\left(\phi \in l_{\infty}^{z}(E)\right)$ with $\|\phi\|_{a, \infty}<\infty$. Observe that $l_{1, \infty}=l_{\infty}$.

A measure is called central if it commutes with all other measures under convolution. One class of central measures are the orbital measures: The orbital measure, $\mu_{g}$, supported on the conjugacy class containing $g \in G$, is the measure defined by

$$
\int_{G} f d \mu_{g}=\int f\left(x g x^{-1}\right) d m_{G}(x) \text { for all continuous functions } f \text { on } G \text {. }
$$

The Fourier transform of a measure $\mu$ is given by $\widehat{\mu}(\sigma)=\int_{G} \sigma d \mu$. A measure is central if and only if $\widehat{\mu}(\sigma)=c_{\sigma} I_{\operatorname{deg}(\sigma)}$ for all $\sigma$ in $\widehat{G}$. Recall that a subset $E$ of $\widehat{G}$ is called a (central) Sidon set if whenever $\left(\phi \in l_{\infty}^{z}(E)\right) \phi \in l_{\infty}(E)$ there is a (central) finite measure $\mu$ such that $\widehat{\mu}(\sigma)=\phi(\sigma)$ for all $\sigma \in E$. Sidon sets in the duals of compact, abelian groups have been extensively studied and found to be very useful and plentiful; indeed, every infinite subset of the dual of a compact, abelian group contains an infinite Sidon set (cf. [13]).

In contrast, compact, simple Lie groups admit no infinite, central Sidon sets [16]. This motivated the introduction of weighted Sidon sets in [8]: A subset $E$ of $\widehat{G}$ is said to be a (central) (a,1)-Sidon set if whenever $\left(\phi \in l_{a, \infty}^{z}(E)\right) \phi \in l_{a, \infty}(E)$ then there is a (central) finite measure $\mu$ such that $\widehat{\mu}(\sigma)=\phi(\sigma)$ for all $\sigma \in E$. (Central) $(1,1)$-Sidon sets are the usual (central) Sidon sets. Weighted Sidon sets can be 
more plentiful. In fact, every infinite subset of the dual of an infinite, compact group contains an infinite, central $(a, 1)$-Sidon set for all $a<1$ [7].

Many Sidon sets in abelian groups have the special property that one can always choose a discrete interpolating measure $\mu$. These sets are known as $I_{0}$ sets and have also been studied by many authors. The result of Hartman and Ryll-Nardzewski implies that every infinite, compact, abelian group admits an infinite $I_{0}$ set (see the remark below). An important open problem is to determine if all Sidon sets are finite unions of $I_{0}$ sets.

It is natural in the non-abelian setting to consider weighted $I_{0}$ sets.

Definition 4.1. We will call a subset $E$ of $\widehat{G}$ an $a-I_{0}$ set if any $\phi \in l_{a, \infty}(E)$ can be interpolated by the Fourier transform of a finite, discrete measure on $G$. It will be called a central $a-I_{0}$ set if any $\phi \in l_{a, \infty}^{z}(E)$ can be interpolated by the Fourier transform of a finite measure that is a linear combination of orbital measures, $\sum b_{k} \mu_{g_{k}}$.

Given the relationship between Sidon and $I_{0}$ sets, it might seem more natural to define a central $a-I_{0}$ set as one for which any $\phi \in l_{a, \infty}^{z}(E)$ could be interpolated by a finite, discrete, central measure. However, not even all finite sets have this property. This is because discrete, central measures on connected groups are supported on the centre of the group [16], and hence any set of cardinality larger than the centre cannot have this alternate property.

However, the expected examples do satisfy our definition.

Proposition 4.1. Any $a-I_{0}$ set is central a-I $I_{0}$. In particular, all finite sets are central $I_{0}$.

Proof. Let $E$ be an $a-I_{0}$ set and assume $\phi \in l_{a, \infty}^{z}(E)$. Choose a finite, discrete measure $\mu=\sum a_{i} \delta_{x_{i}}$ such that $\widehat{\mu}(\sigma)=\phi(\sigma)$ for all $\sigma \in E$ and define $\nu(A)=$ $\int \mu\left(g^{-1} A g\right) d m_{g}$. Then $\nu$ is a measure of norm not exceeding that of $\mu$ and is a sum of orbital measures. Indeed, $\nu=\sum b_{g} \mu_{g}$ where $b_{g}=\sum a_{i}$ with the sum being taken over the indices $i$ such that $x_{i}$ is conjugate to $g$. Moreover, $\widehat{\nu}(\sigma)=\widehat{\mu}(\sigma)=\phi(\sigma)$ for all $\sigma \in E$. Thus $E$ is central $a-I_{0}$.

As with Sidon sets, there are a number of properties equivalent to the definition of central $a-I_{0}$. The following equivalence is relevant for us.

Proposition 4.2. A subset $E \subset \widehat{G}$ is central $a-I_{0}$ if and only if for some $\varepsilon<1$ (equivalently, for every $\varepsilon<1$ ) there is a constant $C$ so that for all choices of $\left\{r_{\sigma}\right\}_{\sigma \in E}, r_{\sigma}= \pm 1$, there is a finite measure $\mu=\sum b_{k} \mu_{g_{k}}$ such that $\|\mu\| \leq C$ and

$$
\sup \left\{(\operatorname{deg} \sigma)^{1-a}\left\|\widehat{\mu}(\sigma)-\frac{r_{\sigma} I_{\operatorname{deg} \sigma}}{(\operatorname{deg} \sigma)^{1-a}}\right\|_{\infty}: \sigma \in E\right\} \leq \varepsilon .
$$

Proof. We need only prove sufficiency, and for this it clearly suffices to interpolate the real-valued sequences in the unit ball of $l_{a, \infty}^{z}(E)$ by finite measures that are linear combinations of orbital measures. In fact, it suffices to show that there is some $\delta<1$ and a constant $C$ such that given any real-valued sequence $\phi$ in the unit ball of $l_{a, \infty}^{z}(E)$ there is a measure $\mu=\sum b_{k} \mu_{g_{k}}$ with $\|\mu\| \leq C, \widehat{\mu}$ real-valued and $\left\|\left.\widehat{\mu}\right|_{E}-\phi\right\|_{a, \infty} \leq \delta$. This is because an iteration argument, such as in [17] Thm. 7 , will then allow us to conclude that interpolation on $E$ can be achieved by a finite measure. This measure, which arises as a limit, will be of the form $\sum b_{k} \mu_{g_{k}}$ 
because the mutual singularity of orbital measures shows that the subspace of finite measures that are linear combinations of orbital measures is norm closed.

So let $\phi$ be a real-valued sequence in the unit ball of $l_{a, \infty}^{z}(E)$, and put $r_{\sigma}=$ $\operatorname{sgn} \phi(\sigma)$. Obtain $\mu$ as in the hypothesis and set $\nu=\mu / 2$. Replacing $\nu$ if necessary by $(\nu+\tilde{\nu}) / 2$, where $\tilde{\nu}(E)=\overline{\nu\left(E^{-1}\right)}$, we can assume $\widehat{\nu}$ is real. It is easy to see that $\left\|\left.\widehat{\nu}\right|_{E}-\phi\right\|_{a, \infty} \leq(1+\varepsilon) / 2=\delta<1$. Moreover, $\nu$ is a linear combination of orbital measures and $\|\nu\| \leq C / 2$.

Remark 4.1. When $G$ is a compact abelian group, the irreducible representations are of degree one. So this proposition implies that the dual of every infinite, compact abelian group admits an infinite $I_{0}$ set. The proposition also shows that Parker's $I$ sets [14] are examples of central $I_{0}$ sets in non-abelian groups.

Since the Fourier transform of any orbital measure satisfies

$$
\widehat{\mu_{g}}(\sigma)=\frac{\operatorname{Tr} \sigma(g)}{\operatorname{deg} \sigma} I_{\operatorname{deg} \sigma},
$$

the results of the previous section have implications for the existence of central $a-I_{0}$ sets. Indeed, Theorems 2.1 and 3.4 together with Proposition 4.2 imply the following statements.

Corollary 4.2.1. (1) If $G$ is any infinite, compact, connected group, then $\widehat{G}$ contains an infinite, central $0-I_{0}$ set.

(2) If $G$ is any compact, simple, simply connected, connected Lie group, other

than $S U(2)$ or $S U(3)$, then $\widehat{G}$ contains an infinite central $a-I_{0}$ set for some $a>0$.

\section{REFERENCES}

[1] H. Bohr, Zur Theorie der Fast Periodischen Funktionen (I. Teil), Acta Math. 45 (1925), 29-127.

[2] H. Bohr, Almost periodic functions (Translated by H. Cohn), Chelsea Publishing Co., New York, 1947. MR 8:512a

[3] T. Bröcker and T. tom Dieck, Representations of compact Lie groups, Graduate Texts in Mathematics, no. 98, Springer-Verlag, New York, 1985. MR 86i:22023

[4] T. Dooley, Central lacunary sets for Lie groups, J. Austral. Math. Soc. Ser. A 45 (1988), 30-45. MR 89j:43007

[5] P. Gallagher, Zeroes of group characters, Math. Z. 87 (1965), 363-364. MR 31:276

[6] K. Hare, The size of characters of compact Lie groups, Studia Mathematica 129 (1998), 1-18. MR 99c:43013

[7] K. Hare, Central Sidonicity for compact Lie groups, Ann. Inst. Fourier (Grenoble) 45 (1995), 547-564. MR 96i:43004

[8] K. Hare and D. Wilson, Weighted p-Sidon sets, J. Austral. Math. Soc. Ser. A 61 (1996), 73-95. MR 97j:43002

[9] S. Hartman and C. Ryll-Nardzewski, Almost periodic extensions of functions, Colloq. Math. 12 (1964), 23-39. MR 29:5057

[10] E. Hewitt and K. Ross, Abstract harmonic analysis II, Springer-Verlag, New York, 1970. MR 41:7378

[11] E. Hewitt and H. Zuckerman, A group theoretic method in approximation theory, Annals of Mathematics 52 (1950), 557-567. MR 12:801c

[12] J. Humphreys, Introduction to Lie algebras and representation theory, Springer-Verlag, New York, 1972. MR 48:2197

[13] J. Lopez and K. Ross, Sidon sets, Lecture Notes in Pure and Applied Math. 13, Marcel Dekker, New York, 1975. MR 55:13173

[14] W. Parker, Central Sidon and central $\Lambda_{p}$ sets, J. Austral. Math. Soc. 14 (1972), 62-74. MR 47:9178 
[15] J. Price, Lie groups and compact groups, London Math. Soc. Lecture Note Series No. 25, Cambridge Univ. Press, Cambridge, 1977. MR 56:8743

[16] D. Ragozin, Central measures on compact simple Lie groups, J. Funct. Anal. 10 (1972), 212-229. MR 49:5715

[17] L. T. Ramsey, Comparisons of Sidon and $I_{0}$ sets, Colloq. Math. 70 (1996), 103-132. MR 97m:43004

[18] D. Rider, Central lacunary sets, Monatsh. Math. 76 (1972), 328-338. MR 51:3801

[19] W. Rudin, Fourier analysis on groups, Interscience Publishers, New York, 1962. MR 27:2808

[20] B. Simon, Representations of finite and compact groups, Graduate Studies in Mathematics 10, Amer. Math. Soc., Providence, RI, 1996. MR 97c:22001

[21] V. Varadarajan, Lie groups, Lie algebras and their representations, Springer-Verlag, New York, 1984. MR 85e:22001

Department of Mathematics and Statistics, University of Missouri-Rolla, Rolla, Missouri 65409

E-mail address: grow@umr.edu

Department of Pure Mathematics, University of Waterloo, Waterloo, Ontario, Canada N2L 3G1

E-mail address: kehare@uwaterloo.ca 